Published in final edited form as:

Am J Obstet Gynecol. 2020 October ; 223(4): 551.e1-551.e7. doi:10.1016/j.ajog.2020.04.006.

\title{
Management of early pregnancy loss with mifepristone and misoprostol: clinical predictors of success from a randomized trial
}

\author{
Sarita SONALKAR, MD, MPH ${ }^{1}$, Nathanael KOELPER, MPH ${ }^{2}$, Mitchell D. CREININ, MD ${ }^{3}$, \\ Jessica M. ATRIO, MD ${ }^{4}$, Mary D. SAMMEL, ScD ${ }^{5}$, Arden McALLISTER, MPH ${ }^{1}$, Courtney A. \\ SCHREIBER, MD, MPH ${ }^{1}$ \\ ${ }^{1}$ Department of Obstetrics and Gynecology, Division of Family Planning, Hospital of the University \\ of Pennsylvania, 3400 Spruce Street, Philadelphia, PA 19104 \\ 2Department of Obstetrics and Gynecology, Center for Research on Reproduction and Women's \\ Health, Philadelphia, PA 19104 \\ ${ }^{3}$ Department of Obstetrics and Gynecology, University of California, Davis; Sacramento, CA \\ ${ }^{4}$ Department of Obstetrics and Gynecology, Montefiore Hospital and Albert Einstein College of \\ Medicine, Bronx, NY \\ ${ }^{5}$ Department of Biostatistics \& Informatics, Center for Integrative Design and Analysis (CIDA), \\ Colorado School of Public Health, Denver, CO
}

\begin{abstract}
Background: Early pregnancy loss (EPL) is a common event in the first trimester, occurring in $15-20 \%$ of recognized pregnancies. A common evidence-based medical regimen for EPL management uses the prostaglandin E1 analogue misoprostol $800 \mathrm{mcg}$ self-administered vaginally. The clinical utility of this regimen is limited by suboptimal effectiveness in women with a closed
\end{abstract}

\footnotetext{
Corresponding Author: Sarita SONALKAR, Department of Obstetrics and Gynecology, Division of Family Planning, Hospital of the University of Pennsylvania, 3400 Spruce Street, Philadelphia, PA 19104, (215) 615-5234, sarita.sonalkar@uphs.upenn.edu. Publisher's Disclaimer: This is a PDF file of an unedited manuscript that has been accepted for publication. As a service to our customers we are providing this early version of the manuscript. The manuscript will undergo copyediting, typesetting, and review of the resulting proof before it is published in its final form. Please note that during the production process errors may be discovered which could affect the content, and all legal disclaimers that apply to the journal pertain.

Disclosures:

SONALKAR. The author reports no conflicts of interest.

KOELPER. The author reports no conflicts of interest.

CREININ. The author is a consultant for Danco Laboratories.

ATRIO. The author reports no conflicts of interest.

SAMMEL. The author reports no conflicts of interest.

SCHREIBER. The author is a consultant for Danco Laboratories.

Clinical trial information:

This trial was registered with Clinicaltrials.gov, protocol number NCT02012491.

Paper presentation information:

We presented these data as a poster abstract at the American Society for Reproductive Medicine 2019 Scientific Congress on October $15^{\text {th }}, 2019$ in Philadelphia, PA.

Authors employed by the Federal Government or Armed Forces:

None.
} 
cervical os, with $29 \%$ of women with EPL requiring a second dose after three days, and $16 \%$ eventually requiring a uterine aspiration procedure.

Objectives: To evaluate characteristics associated with treatment success in women receiving medical management with mifepristone-misoprostol or misoprostol alone for early pregnancy loss (EPL).

Study Design: We performed a planned secondary analysis of a randomized trial comparing mifepristone-misoprostol to misoprostol alone for EPL treatment. The published prediction model for success of single-dose vaginal misoprostol included the following variables: active bleeding, type of EPL (anembryonic pregnancy or embryonic/fetal demise), parity, gestational age, and treatment site; previous significant predictors were vaginal bleeding within the past 24 hours, and parity of 0 or 1 versus higher. We first assessed in bivariate analyses if these characteristics predicted differential proportions of women with success or failure; given the small proportion of treatment failures in the combined treatment arm, both arms were combined for analysis. We then performed a logistic regression analysis to assess the effect of these factors collectively in each of the two treatment groups separately as well as in the full cohort as a proxy for the combined treatment arm. We tested the ability of characteristics previously associated with misoprostol success to discriminate successful from failed treatment using receiver-operating characteristic curves. We calculated the area under the curve (AUC) to quantify the ability of the score to discriminate between treatment success or failure in each treatment arm as well as in the entire cohort. Using multivariable logistic regression, we then assessed our study population for other predictors of treatment success in both treatment groups, with and without mifepristone.

Results: This analysis includes all 297 evaluable subjects in the primary study, including 148 in the mifepristone-misoprostol combined and 149 in the misoprostol-alone groups. Among women who had vaginal bleeding at the time of treatment, $15 / 17$ (88\%) in the mifepristone-misoprostol combined group and 12/17 (71\%) of those in the misoprostol-alone group expelled the pregnancy. Among women with a parity of 0 or 1,94/108 (87\%) in the mifepristone-misoprostol combined group, and 66/95 (69\%) of those in the misoprostol-alone group expelled the pregnancy. These clinical characteristics did not predict success above chance alone in the combined cohort (AUC $=0.56,95 \%$ CI 0.48-0.64). No other baseline clinical factors predicted treatment success in the misoprostol-alone or mifepristone pretreatment arms individually. In the full cohort, the only significant predictors of treatment success were mifepristone pretreatment (aOR 2.51, 95\% CI 1.43-4.43), and smoking (aOR 2.15, 95\% CI 1.03-4.49).

Conclusion: No baseline clinical factors predict success in women undergoing medical management of EPL with misoprostol. Adding mifepristone to the EPL medical management regimen improves treatment success and should be used regardless of baseline clinical characteristics.

\section{Keywords}

early pregnancy loss; medical management; mifepristone; miscarriage; misoprostol 


\section{Introduction}

Early pregnancy loss (EPL) is a common event in the first trimester of pregnancy, occurring in 15-20\% of recognized pregnancies (1). Both providers and patients have shown an interest in pursuing nonsurgical treatment options for EPL (2). A common evidence-based EPL medical management regimen uses the prostaglandin E1 analogue misoprostol $800 \mathrm{mcg}$ self-administered vaginally to facilitate pregnancy tissue expulsion (3-5). The clinical utility of this regimen is limited by suboptimal effectiveness in women with a closed cervical os (6), with $29 \%$ of women with EPL requiring a second treatment dose after three days and $16 \%$ eventually requiring a uterine aspiration procedure $(3,7)$.

In 2018, we reported the results of a multicenter trial designed to evaluate if mifepristone pretreatment could improve misoprostol effectiveness (8). We included 297 women with anembryonic gestation or embryonic/fetal demise to receive misoprostol vaginally with or without mifepristone pretreatment; treatment success (complete pregnancy expulsion) rates with one misoprostol dose and mifepristone pretreatment (84\%, 95\% CI 77-90\%) was higher than with misoprostol alone $(67 \%, 95 \%$ CI 59-75\%)(8). Unfortunately, these positive findings may not translate to a shift in current clinical care in the U.S. because mifepristone access is restricted under current FDA requirements, making mifepristone difficult to access in many locations (9). Accordingly, we sought to identify characteristics within our study population that could be predictive of improved success for women who may be offered misoprostol alone.

A secondary analysis of a U.S. multicenter study performed in the mid-2000s identified basic clinical characteristics that predicted treatment success with EPL medical management from 5-12 weeks gestational age (7). The primary predictors demonstrated in this model, reported in 2006, were vaginal bleeding and parity of 0 or 1 . Our primary objective was to evaluate if these previously identified clinical characteristics are associated with greater success in the misoprostol-alone arm of our trial. In addition, we sought to identify characteristics that predict success in each arm of the study and in the combined cohorts to help inform treatment decision making for women deciding between medical and surgical EPL management.

\section{Materials and Methods}

We performed this planned secondary analysis to evaluate clinical predictors previously associated with single-dose vaginal misoprostol EPL treatment success (7), with and without mifepristone pretreatment. The results of the primary study of EPL medical management have been previously reported (8). In brief, we enrolled 300 women in a multi-center, randomized, single-masked trial to compare the effectiveness of combination treatment (mifepristone $200 \mathrm{mg}$ orally followed 24 hours later by misoprostol $800 \mathrm{mcg}$ vaginally) to usual treatment (misoprostol $800 \mathrm{mcg}$ vaginally). The final evaluable cohort included 148 and 149 women in the two treatment groups, respectively. The trial included women 18 years and older diagnosed with a nonviable intrauterine pregnancy (anembryonic gestation or embryonic/fetal demise) between 5 and 12 weeks gestation, and excluded women with an incomplete or inevitable abortion, and women clinically ineligible for EPL medical 
management (8). Participants were recruited from a range of practice settings, including those offering providing services in obstetrics and gynecological services and primary care services (Table 1). The primary outcome was complete expulsion of the gestational sac by the first follow-up visit (24h after misoprostol use, range days 2-5) without further intervention over the 30-day study period. Women who did not expel the gestational sac could opt for a second misoprostol dose, surgical aspiration or expectant management. The trial was registered with Clinicaltrials.gov, protocol number NCT02012491. The primary study had greater than $90 \%$ power to detect a ratio of 2 for the risk of failure in the mifepristone pretreatment arm compared to the misoprostol-alone arm.

For this analysis, we first attempted to validate previously described predictors of success of medical management of EPL with a single dose of vaginal misoprostol alone. The published prediction model (7) for single-dose vaginal misoprostol included the following variables: active bleeding, type of EPL (anembryonic pregnancy or embryonic/fetal demise), parity, gestational age, and treatment site; previous significant predictors were vaginal bleeding within the past 24 hours, and parity of 0 or 1 versus higher. We hypothesized that the sensitivity of the combined predictive markers to predict success would be $90 \%+/-5 \%$.

To apply the previously published prediction rule to our population, we computed a weighted score by using the log-odds ratios of each predictor listed in the published multivariable model (active bleeding, type of EPL, parity, gestational age, and treatment site). We summed risk factor weights for each subject, based on whether or not the individual participant possessed the clinical characteristic(s). We created receiver operating characteristic curves (ROC) and calculated the area under the curve (AUC) to quantify the ability of the score to discriminate between treatment success or failure in each arm as well as in the entire cohort. The AUC is a summary of diagnostic accuracy: if the AUC equals 0.5 , the ROC curve corresponds to random chance; if the AUC equals 1, the diagnostic model has perfect accuracy (10). We grouped the scores into deciles, to investigate differences in success by summed weights and to assess goodness-of-fit. We used logistic regression to predict the probability of successful management based on score decile (11).

Next, we assessed in bivariate analyses if these characteristics predicted differential proportions of women with success or failure using Pearson $\chi^{2}$ analyses. Given the small proportion of treatment failures in the combined treatment arm, the arms were combined for analysis. We then performed a logistic regression analysis to assess the effect of these factors collectively in each of the two treatment groups separately as well as in the full cohort as a proxy for the combined treatment arm.

Lastly, we assessed the remaining clinical predictors of success of medical management of EPL in the full cohort of participants (who used misoprostol with or without mifepristone), as well as in each of the treatment arms separately. We performed bivariate analyses using Pearson $\chi 2$ analyses or Wilcoxon rank sum tests as appropriate, comparing women in the full cohort of participants who had success or failure of medical management of EPL, by demographic and clinically relevant factors. We evaluated treatment success in a multivariable logistic regression analysis by performing stepwise backwards selection for any covariates from Table 1 with a $\mathrm{P} \$ \mathbf{\$} .2$ and the set of 2006 predictors (11). 


\section{Results}

This analysis includes all 297 evaluable subjects in the primary study, including 148 in the mifepristone-misoprostol combined treatment and 149 in the misoprostol-alone groups. Bivariate analysis of predictors of success for the full cohort are presented in Table 1. Using the combined predictive variables of vaginal bleeding and parity of 0 or 1 , we had $90 \%+l$ $-3 \%$ power to detect success with $90 \%$ sensitivity.

Previously described predictors of success of medical management with misoprostol did not differ by randomization group (Table 2). When we applied the predictors to our population using risk factor weights to create a risk score, the odds ratio for increased success by decile in the full cohort was 1.08 (95\% CI 0.98, 1.18; Figure 1). The area under the receiver operating characteristics curve using the score based on the predictors was $0.56(95 \% \mathrm{CI}$ 0.48-0.64) in the full cohort (Figure 1).

Bivariate predictors of medical management success in the full cohort included non-smoker status ( $\mathrm{p}=0.01$ ), pain during periods $(\mathrm{p}=0.19)$, and randomization group $(\mathrm{p}=0.001$; Table 1$)$. In the multivariable logistic regression model, both mifepristone pretreatment $(\mathrm{P}=0.001)$ and non-smoking status $(\mathrm{p}=0.04)$ remained significant in the full cohort. However, non-smoking status was not significant in the model for the misoprostol-alone group $(\mathrm{p}=0.06)$ or mifepristone pretreatment group $(\mathrm{p}=0.44)$. The area under the receiver operating characteristics curve was 0.64 (95\% CI 0.56-0.7) for the full cohort.

\section{Discussion}

\section{Principal findings}

In this planned secondary analysis of a randomized controlled trial comparing the efficacy of pretreatment with mifepristone followed by misoprostol versus misoprostol alone for EPL management, we found no clinical or medical history predictors of treatment success, except for nonsmoking status. When restricting our analysis to the treatment group that received misoprostol alone (the treatment group that might benefit most from a described "phenotype" for success), previously described clinical predictors for success, parity and current bleeding, did not predict success.

\section{Results in context}

We modeled this research on a prior U.S. multicenter study of clinical predictors for success in a population of 491 women who received misoprostol alone for EPL management (7). In that study, authors found that vaginal bleeding within the past 24 hours and nulliparity or low parity predicted success with a single misoprostol dose. Nulliparous or primiparous women with bleeding in the preceding 24 hours had success rates of $79 \%$ and $77 \%$, respectively. Of note, overall success of medical management of EPL (including up to 2 doses of misoprostol up to 30 days after initial management), was $95 \%$ in women who had lower abdominal pain and bleeding in the past 24 hours [7]. Our current study was focused on assessing treatment success after one misoprostol dose in accordance with patient preferences [2]; we did not identify clinical characteristics associated with successful expulsion in either the misoprostol-only or mifepristone pretreatment arms. 
Our inability to validate previously determined predictors of treatment success may be partially attributable to differences in the study populations. The study sites differed from the 2006 study that included 4 sites all on the United States east coast (New York, Philadelphia, Pittsburgh and Miami) (7), while our current study included subjects from New York, Philadelphia and Sacramento, with $26 \%$ of participants from California (8). However, the proportion of women with treatment success in each group in our study did not vary by site. Perhaps more important are differences in the presence of bleeding between the two studies. In the 2006 study (1), $64 \%$ had vaginal bleeding within the 24 hours prior to treatment and $88 \%$ of these women with vaginal bleeding had success with up to 2 doses of misoprostol. In our study, only $12 \%$ of women had any bleeding prior to randomization (8). It is possible that misoprostol alone is an appropriate treatment regimen for women with EPL who are already having bleeding, but the small proportion of women with bleeding in our study diminished our ability to recognize this association. Alternatively, pretreatment with mifepristone in a population of women who are already bleeding is unlikely to have adverse effects and may improve success rates.

\section{Clinical and research implications}

In our population, self-reported non-smoking status predicted treatment success in the full cohort, although this risk factor did not achieve significance in either group separately. The reason for this finding is unclear and should be interpreted with caution; the association was based on a small cohort of smokers (13\% of the total population) and could represent some other unmeasured variable. Chronic nicotine may decrease uterine blood flow (12), and can prolong gestation and inhibit cervical ripening in rats, possibly by suppression of an antiinflammatory response (13). The pathophysiology of this pathway in humans is not elucidated. The 2006 study did not include smoking in its assessment of clinical predictors of success. Smoking prevalence has decreased in the United States (14) but remains prevalent in other countries (15); the interplay between smoking and EPL management strategies may deserve further study.

\section{Strength and limitations}

The strength of this planned secondary analysis includes its diverse population with prospective data collection from a randomized controlled trial. We were limited by the small proportion of treatment failures in the mifepristone pretreatment group. Although we analyzed for baseline clinical predictors for success in this group, a larger sample size would have allowed for more power to detect individual predictors. Our study sample had differing clinical characteristics as compared with the 2006 comparison study, which may have affected the validation of prior predictors of treatment success with misoprostol alone. Future cohort studies examining a larger population of women receiving combined treatment with mifepristone and misoprostol for EPL may identify important baseline clinical predictors for treatment success.

\section{Conclusion}

In summary, we found that previously described clinical predictors do not support large effects of particular patient characteristics having similar success using misoprostol without mifepristone pretreatment, nor we were able to identify additional baseline clinical factors 
that would support the use of misoprostol without mifepristone for EPL management. Given the improvement in success with mifepristone pretreatment discovered in the primary study, the results of this secondary analysis further support the recommendation that all women who desire misoprostol management of EPL should receive pretreatment with mifepristone to maximize the likelihood of success.

\title{
Acknowledgements
}

Supported by the National Institute of Child Health and Human Development of the National Institutes of Health (Eunice Kennedy Shriver award number R01-HD0719-20 [to Dr. Schreiber] and Women's Reproductive Health Research award number K12-HD001265-19 [to Dr. Sonalkar]), and a Society of Family Planning Research Fund Midcareer Mentor Award (Schreiber). Paul Whittaker, DPhil, provided assistance in manuscript writing.

\begin{abstract}
Supported by the National Institute of Child Health and Human Development of the National Institutes of Health (Eunice Kennedy Shriver award number R01-HD0719-20 [to Dr. Schreiber] and Women's Reproductive Health Research award number K12-HD001265-19 [to Dr. Sonalkar]), and a Society of Family Planning Research Fund Midcareer Mentor Award (Schreiber). Dr. Creinin has served as a consultant for Danco. The other authors did not report any other potential conflicts of interest.
\end{abstract}

Financial support for the research:

Supported by the National Institute of Child Health and Human Development of the National Institutes of Health (Eunice Kennedy Shriver award number R01-HD0719-20 [to Dr. Schreiber] and Women's Reproductive Health Research award number K12-HD001265-19 [to Dr. Sonalkar]), and a Society of Family Planning Research Fund Midcareer Mentor Award (Schreiber).

\section{References}

1. Ventura SJ, Curtin SC, Abma JC, Henshaw SK. Estimated pregnancy rates and rates of pregnancy outcomes for the United States, 1990-2008. Natl Vital Stat Rep. 2012;60(7):1-21.

2. Schreiber CA, Chavez V, Whittaker PG, Ratcliffe SJ, Easley E, Barg FK. Treatment Decisions at the Time of Miscarriage Diagnosis. Obstet Gynecol. 2016;128(6):1347-56. [PubMed: 27824749]

3. Zhang J, Gilles JM, Barnhart K, Creinin MD, Westhoff C, Frederick MM, et al. A comparison of medical management with misoprostol and surgical management for early pregnancy failure. $\mathrm{N}$ Engl J Med. 2005;353(8):761-9. [PubMed: 16120856]

4. Gemzell-Danielsson K, Bygdeman M, Aronsson A. Studies on uterine contractility following mifepristone and various routes of misoprostol. Contraception. 2006;74(1):31-5. [PubMed: 16781257]

5. ACOG Practice Bulletin No. 200: Early Pregnancy Loss. Obstet Gynecol. 2018;132(5):e197-e207. [PubMed: 30157093]

6. Neilson JP, Hickey M, Vazquez J. Medical treatment for early fetal death (less than 24 weeks). Cochrane Database Syst Rev. 2006(3):CD002253. [PubMed: 16855990]

7. Creinin MD, Huang X, Westhoff C, Barnhart K, Gilles JM, Zhang J, et al. Factors related to successful misoprostol treatment for early pregnancy failure. Obstet Gynecol. 2006;107(4):901-7. [PubMed: 16582130]

8. Schreiber CA, Creinin MD, Atrio J, Sonalkar S, Ratcliffe SJ, Barnhart KT. Mifepristone Pretreatment for the Medical Management of Early Pregnancy Loss. N Engl J Med. 2018;378(23):2161-70. [PubMed: 29874535]

9. Mifeprex (mifepristone) Tablets, 200mg Risk Evaluation and Mitigation Strategy. U.S. Food \& Drug Administration; 2016.

10. Zou KH, O’Malley AJ, Mauri L. Receiver-operating characteristic analysis for evaluating diagnostic tests and predictive models. Circulation. 2007;115(5):654-7. [PubMed: 17283280]

11. Moons KG, Altman DG, Reitsma JB, Ioannidis JP, Macaskill P, Steyerberg EW, et al. Transparent Reporting of a multivariable prediction model for Individual Prognosis or Diagnosis (TRIPOD): explanation and elaboration. Ann Intern Med. 2015;162(1):W1-73. [PubMed: 25560730] 
12. Lambers DS, Clark KE. The maternal and fetal physiologic effects of nicotine. Semin Perinatol. 1996;20(2):115-26. [PubMed: 8857697]

13. Yang J, Shi SQ, Shi L, Liu H, Fang D, Garfield RE. Nicotine treatment prolongs gestation and inhibits cervical ripening in pregnant rats. Am J Obstet Gynecol. 2014;210(1):76e1-7. [PubMed: 24036401]

14. Current Cigarette Smoking Among Adults-United States, 2017. Centers for Disease Control and Prevention; 2018 Contract No.: 44.

15. WHO global report on trends in prevalence of tobacco smoking. Geneva, Switzerland: World Health Organization; 2015. 


\section{Condensation:}

When evaluating predictors of misoprostol early pregnancy loss treatment success, mifepristone pretreatment is a better predictor than baseline clinical factors, including vaginal bleeding or parity. 


\section{AJOG at a Glance:}

A. Why was this study conducted?

- To evaluate characteristics associated with treatment success in women receiving medical management of early pregnancy loss (EPL).

B. What are the key findings?

- Mifepristone pretreatment and nonsmoking status were the only predictors of treatment success in our population

- $\quad$ Previously described clinical predictors of success with misoprostol alone were not validated in our population, nor did we identify important clinical factors that would support the use of misoprostol without mifepristone for EPL management.

C. What does this study add to what is already known?

- We evaluated previously described predictors of EPL medical treatment success in a diverse cohort, including patients receiving mifepristone pretreatment.

- $\quad$ Pretreatment with mifepristone is a more useful intervention than considering baseline clinical characteristics to maximize treatment success in women undergoing misoprostol treatment of EPL. 


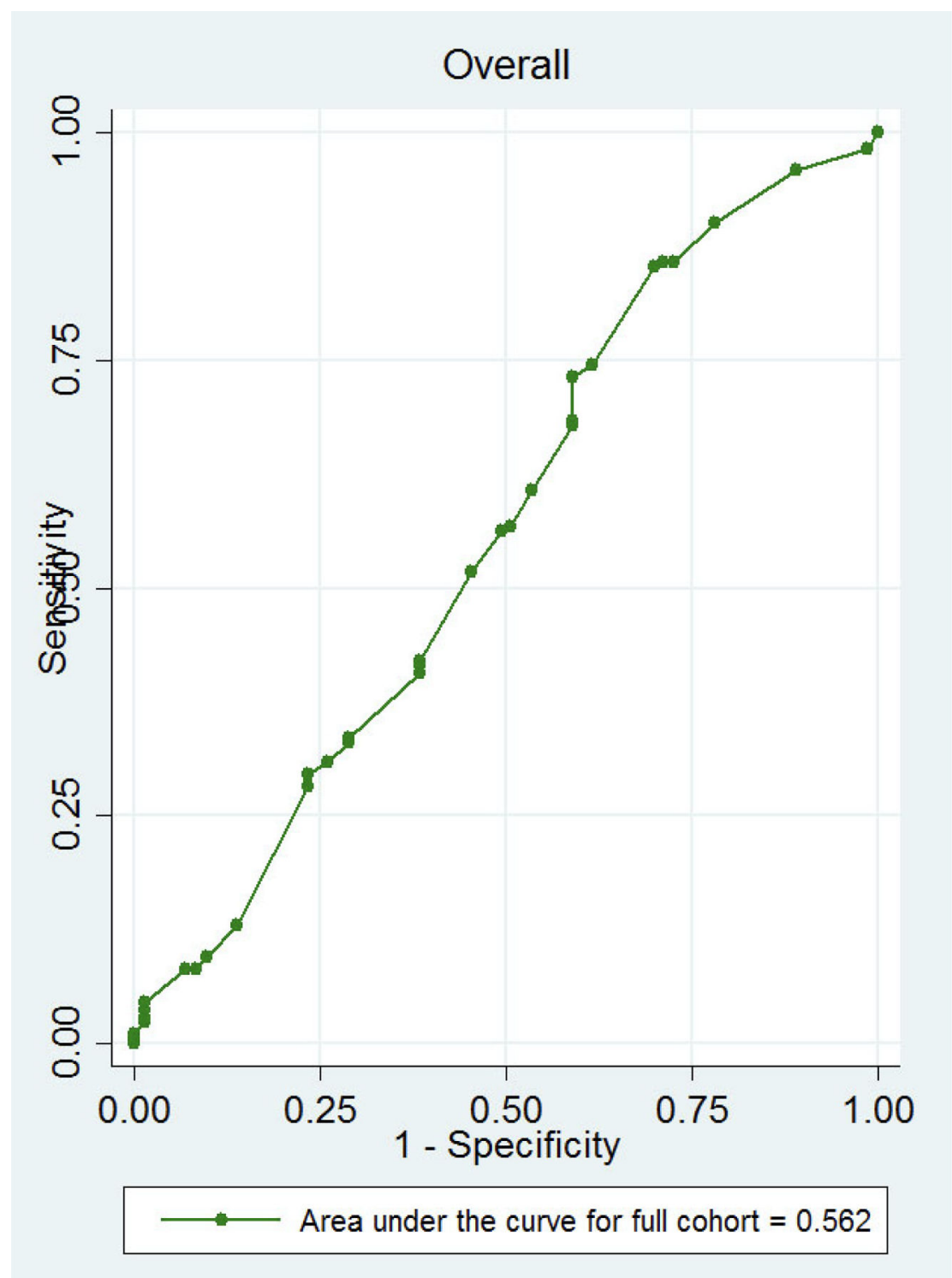

Receiver operating characteristics curve of success using the 2006 model

Receiver operating characteristics curve for the full cohort (AUC 0.56 95\% CI 0.48-0.64) applying the 2006 predictor model for single-dose misoprostol success of medical management of EPL. 95\% confidence interval contains 0.5 and thus the test is no different than random chance. (7) 
Table 1:

Demographic and clinical characteristics by failure or success of medical management of early pregnancy loss

\begin{tabular}{|c|c|c|c|c|}
\hline & Full cohort N=297 & Failure $n=73$ & Success $n=224$ & p-value \\
\hline Median age (years) & $31(26-35)$ & $30(25-35)$ & $31(26-35)$ & 0.5 \\
\hline Mean BMI $\left(\mathrm{kg} / \mathrm{m}^{2}\right)$ & $27.4(23.2-32.5)$ & $27.8(23.8-32.3)$ & $27.3(23.0-32.7)$ & 0.64 \\
\hline Race & & & & 0.60 \\
\hline Black or African American & $131(44)$ & $38(29)$ & $93(71)$ & \\
\hline White & $108(36)$ & $25(23)$ & $83(77)$ & \\
\hline Mixed/more than one race & $30(10)$ & $6(20)$ & $24(80)$ & \\
\hline Asian & $20(7)$ & $3(15)$ & $17(85)$ & \\
\hline Native Hawaiian/Pacific Islander & $2(1)$ & $0(0)$ & $2(100)$ & \\
\hline Other/unknown & $6(2)$ & $1(117)$ & $5(83)$ & \\
\hline Ethnicity & & & & 0.51 \\
\hline Non-Hispanic or Non-Latina & $219(74)$ & $56(26)$ & $163(74)$ & \\
\hline Hispanic or Latina & $78(26)$ & $17(22)$ & $61(78)$ & \\
\hline Smoking ${ }^{*}$ & & & & 0.01 \\
\hline No & $259(87)$ & $57(22)$ & $202(78)$ & \\
\hline Yes & $37(13)$ & $15(41)$ & $22(59)$ & \\
\hline Prior early pregnancy loss & & & & 0.87 \\
\hline No & $193(65)$ & $48(25)$ & $145(75)$ & \\
\hline Yes & $104(35)$ & $25(24)$ & $79(76)$ & \\
\hline Prior induced abortion & & & & 0.40 \\
\hline No & $199(67)$ & $46(23)$ & $153(77)$ & \\
\hline Yes & $98(33)$ & $27(28)$ & $71(72)$ & \\
\hline Prior medical abortion ${ }^{*}$ & & & & 0.23 \\
\hline No & $274(93)$ & $69(25)$ & $205(75)$ & \\
\hline Yes & $22(7)$ & $3(1)$ & $19(86)$ & \\
\hline Prior surgical abortion ${ }^{*}$ & & & & 0.21 \\
\hline No & $202(68)$ & $45(23)$ & $157(78)$ & \\
\hline Yes & $93(32)$ & $27(29)$ & $66(71)$ & \\
\hline Parity & & & & 0.27 \\
\hline 0 & $114(38)$ & $24(21)$ & $90(79)$ & \\
\hline 1 or more & $183(62)$ & $49(27)$ & $134(73)$ & \\
\hline Pain during periods & & & & 0.19 \\
\hline No pain & $56(19)$ & $21(38)$ & $35(62)$ & \\
\hline Very little & $76(26)$ & $14(18)$ & $62(82)$ & \\
\hline Some & $84(28)$ & $20(24)$ & $64(76)$ & \\
\hline Quite a bit & $35(12)$ & $9(26)$ & $26(74)$ & \\
\hline Very much & $39(13)$ & $8(21)$ & $31(79)$ & \\
\hline
\end{tabular}




\begin{tabular}{|c|c|c|c|c|}
\hline & Full cohort $\mathrm{N}=297$ & Failure $n=73$ & Success $n=224$ & p-value \\
\hline Worst pain & $7(2)$ & $1(14)$ & $6(86)$ & \\
\hline Gestational age & & & & 0.75 \\
\hline$<7$ Weeks & $107(36)$ & $27(25)$ & $80(75)$ & \\
\hline 7-8 6/7 Weeks & $144(48)$ & $33(23)$ & $111(77)$ & \\
\hline $9-126 / 7$ Weeks & $46(15)$ & $13(28)$ & $33(72)$ & \\
\hline Diagnosis & & & & 0.52 \\
\hline Embryonic/fetal demise & $220(74)$ & $52(24)$ & $168(76)$ & \\
\hline Anembryonic gestation & $77(26)$ & $21(27)$ & $56(73)$ & \\
\hline Method of pregnancy conception & & & & 0.13 \\
\hline Spontaneous & $276(94)$ & $71(26)$ & $205(74)$ & \\
\hline Assisted reproductive technologies & $16(5)$ & $1(6)$ & $15(94)$ & \\
\hline Active bleeding & & & & 0.74 \\
\hline No & $288(77)$ & $56(25)$ & $172(75)$ & \\
\hline Yes & $34(11)$ & $7(21)$ & $27(79)$ & \\
\hline Not assessed & $35(12)$ & $10(29)$ & $25(71)$ & \\
\hline Rh status & & & & 0.94 \\
\hline $\mathrm{Rh}-$ & $24(8)$ & $6(25)$ & $18(75)$ & \\
\hline $\mathrm{Rh}+$ & $268(92)$ & $65(24)$ & $203(76)$ & \\
\hline Uterine tenderness ${ }^{*}$ & & & & 0.80 \\
\hline No & 257 (87) & $62(24)$ & $195(76)$ & \\
\hline Yes & $11(4)$ & $3(27)$ & $8(73)$ & \\
\hline Not assessed & $27(9)$ & $8(30)$ & $19(70)$ & \\
\hline Randomization arm & & & & 0.001 \\
\hline Misoprostol alone & $149(50)$ & $49(33)$ & $100(67)$ & \\
\hline Mifepristone pretreatment & $148(50)$ & $24(16)$ & $124(84)$ & \\
\hline Site & & & & 0.099 \\
\hline University of Pennsylvania & $160(54)$ & $47(29)$ & $113(71)$ & \\
\hline University of California, Davis & $76(26)$ & $13(17)$ & $63(83)$ & \\
\hline Albert Einstein College of Medicine & $61(21)$ & $13(21)$ & $48(79)$ & \\
\hline
\end{tabular}

Data are presented as n (\%), mean (standard deviation), or median (interquartile range). Column percentages are presented for the full cohort; row percentages are presented otherwise.

* Data missing for Smoking ( $\mathrm{n}=1)$, Prior medical abortion $(\mathrm{n}=1)$, Prior surgical abortion $(\mathrm{n}=2)$, Rh status $(\mathrm{n}=5)$, Uterine tenderness $(\mathrm{n}=2)$, Method of pregnancy conception (3) 
Table 2:

Distribution by treatment group of variables included in the previously-described predictor model ${ }^{*}$ for singledose misoprostol success of early pregnancy loss management

\begin{tabular}{|c|l|l|l|l|}
\hline & Full cohort & Misoprostol alone & Mifepristone pretreatment & p-value \\
\hline Active bleeding & & & & 0.65 \\
\hline No & $288(77)$ & $117(79)$ & $111(75)$ & \\
\hline Yes & $34(11)$ & $17(11)$ & $17(11)$ & \\
\hline Not Assessed & $35(12)$ & $15(10)$ & $20(14)$ & \\
\hline Diagnosis & & & & 0.67 \\
\hline Embryonic/fetal demise & $220(74)$ & $112(75)$ & $108(73)$ & \\
\hline Anembryonic gestation & $77(26)$ & $37(25)$ & $40(27)$ & \\
\hline Parity & & & & 0.19 \\
\hline 0 & $114(38)$ & $51(34)$ & $63(43)$ & \\
\hline 1 & $89(30)$ & $44(30)$ & $45(30)$ & \\
\hline $2+$ & $94(32)$ & $54(36)$ & $40(27)$ & 0.75 \\
\hline Gestational age & & & & \\
\hline$<7$ Weeks & $107(36)$ & $27(37)$ & $80(36)$ & \\
\hline $7-86 / 7$ Weeks & $144(48)$ & $33(45)$ & $111(50)$ & \\
\hline $9-12$ 6/7 Weeks & $46(15)$ & $13(18)$ & $33(15)$ & \\
\hline Site & & & & $80(54)$ \\
\hline University of Pennsylvania & $160(54)$ & $80(54)$ & $38(26)$ & \\
\hline University of California, Davis & $76(26)$ & $38(26)$ & $30(20)$ & \\
\hline Albert Einstein College of Medicine & $61(21)$ & $31(21)$ & & \\
\hline
\end{tabular}

Data are presented as $\mathrm{n}(\%)$.

from Creinin MD, Huang X, Westhoff C, Barnhart K, Gilles JM, Zhang J, et al. Factors related to successful misoprostol treatment for early pregnancy failure. Obstet Gynecol. 2006;107 (4):901-7. 
Table 3:

Final multivariable model for within-study clinical predictors of success of medical management of early pregnancy loss

\begin{tabular}{|l|l|l|l|l|l|l|}
\hline & OR & 95\% CI & p-value & aOR* & 95\% CI & p-value \\
\hline Smoking & & & & & & \\
\hline Yes & referent & & & referent & & \\
\hline No & 2.41 & $1.18-4.96$ & 0.02 & 2.15 & $1.03-4.49$ & 0.04 \\
\hline Randomization arm & & & & & & \\
\hline Misoprostol alone & referent & & & referent & & \\
\hline Mifepristone pretreatment & 2.53 & $1.45-4.41$ & 0.001 & 2.51 & $1.43-4.43$ & 0.001 \\
\hline
\end{tabular}

* Adjusted for smoking and treatment arm

OR: odds ratio; CI: confidence interval; aOR: adjusted odds ratio 